\title{
INVESTIGACIÓN EN DISEÑO EN
}

TIEMPOS DE PANDEMIA:

RELACIONES ENTRE REFLEXIÓN Y

ACCIÓN

\author{
DESIGN RESEARCH IN \\ PANDEMIC TIMES: \\ RELATIONS BETWEEN THOUGHT \\ AND ACTION
}

\section{María Camila}

\section{Rodríguez Arango}

Grupo de investigación

Pensamiento de Diseño,

Semillero 3E, Diseño de

Espacios y Escenarios,

Universidad Piloto de

Colombia.

maria-rodriguez6@upc.

edu.co

Jessica Vargas Sanabria

Grupo de investigación

Pensamiento de Diseño,

Semillero 3E, Diseño de

Espacios y Escenarios,

Universidad Piloto de

Colombia.

jvargas70@upc.edu.co

\section{Andrés Vicente}

Colorado Bonilla

Grupo de investigación

Pensamiento de Diseño

- Semillero 3E, Diseño de

Espacios y Escenarios,

Universidad Piloto de

Colombia.

andres-colorado@upc.

edu.co

Renata Marcela Gómez

\section{Sandoval}

Grupo de investigación

Pensamiento de Diseño,

Semillero 3E, Diseño de

Espacios y Escenarios,

Universidad Piloto de

Colombia.

renata-gomez@upc.

edu.co
Camilo Ernesto Fuentes

Arciniegas

Grupo de investigación

Pensamiento de Diseño,

Semillero 3E, Diseño de

Espacios y Escenarios,

Universidad Piloto de

Colombia.

camilo-fuentes@upc.

edu.co

\section{Jorge Arturo González}

\section{Castro}

Grupo de investigación

Pensamiento de Diseño,

Semillero 3E, Diseño de

Espacios y Escenarios,

Universidad Piloto de

Colombia.

jorge-gonzalez@upc.

edu.co 
Palabras clave

Diseño, espacios,

escenarios, metodología,

investigación,

pensamiento de diseño,

virtualidad.

\section{Resumen}

Los procesos de investigación

formativa en diseño, al igual que muchas de las esferas de la vida académica, se vieron afectados

por la emergencia sanitaria del Covid-19 durante el año 2020. Esto conlleva a diferentes procesos de adaptación que modificaron las lógicas de investigación estructuradas desde un principio. En consecuencia, los estudiantes y los docentes tuvieron que replantear las interacciones que demanda un proyecto de impacto social bajo estas condiciones. Es por ello que este artículo tiene por objeto relacionar las experiencias de investigación-creación que se desarrollaron en el Semillero de Investigación en Diseño 3E del programa de Diseño de Espacios y Escenarios de la Universidad Piloto de Colombia, mediante

la implementación de una

estrategia de diseño dirigida a los pobladores del corregimiento de Palermo, perteneciente al municipio de Paipa en Boyacá. La finalidad del proyecto se traduce en salvaguardar y apropiar el patrimonio cultural de su territorio. Es así cómo se generó un escenario de aprendizaje y co-creación en el que por medio de lecturas de contexto se han logrado reconocer e interpretar los procesos de reflexión y acción desde estructuras de pensamiento y herramientas de diseño, lo que permite evidenciar una estructura metodológica de acción proyectual en diseño con impacto social mediada por la virtualidad, que abre una discusión sobre cómo investigar en diseño de manera remota.

a

\section{Abstract}

Formative research processes in design, like many of the spheres of academic life, were affected by the Covid-19 health emergency during 2020. This led to a different process of adaptation that modified the research of logics structure from beginning. Consequently, students and teachers had to rethink the interactions that a social impact project demands under these conditions. That is why this article aims to relate the research-creation experiences that were developed in the 3E Design Research Seedbed of the Design of Spaces and Scenarios program of the Pilo University of Colombia, through the implementation of a design strategy addressed to the residents of the town of Palermo, belonging to the municipality of Paipa in
Boyacá. The purpose of the project is to safeguard and appropriate the cultural heritage of its territory. This is how a learning and cocreation scenario was generated in which, through context readings, the processes of reflection and action have been recognized and interpreted from thought structures and design tools, which allows to demonstrate a methodological structure of action project in design with social impact mediated by virtuality, which opens a discussion on how to research design remotely. 
la población juvenil sobre el valor identitario del patrimonio material e inmaterial palermano, lo que podría ser un factor importante en el aparente desarraigo al territorio que ubica sus proyectos de vida fuera

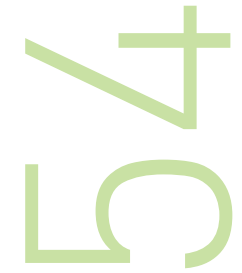
de Palermo. Algo en cierta medida propiciado por una concentración de dicho capital sociocultural en los adultos mayores, quienes reconocen el valor de Palermo en sus narraciones autobiográficas (experiencias personales), pero no las hacen circular entre los pobladores. Una posible consecuencia sería el desencuentro generacional de estos con los palermanos jóvenes, dando lugar a una hipótesis de investigación en torno a una pregunta: ¿Cómo incentivar la apropiación del patrimonio cultural en la comunidad del corregimiento de Palermo?

Este artículo recoge las experiencias de este proceso de rieren una cienta complejdad a causa de una general refieren una cierta complejidad a causa de una generalizada separación entre lo que implica investigar y diseñar. Algo que, en el caso particular del proceso formativo de investigación en diseño requiere orientar acciones para desarrollar experiencias de aprendizaje en las que, sin perder el rigor metodológico de la investigación, se privilegie el desarrollo de proyectos de diseño con el objeto de producir, validar y sistematizar conocimiento, para que sea transferible y aplicable a una realidad social.

Consciente de esto, el programa de Diseño de Espacios y Escenarios de la Universidad Piloto de Colombia, desde el año 2018 adelanta esfuerzos orientados a la conformación y consolidación de una línea de investigación denominada Pensamiento de Diseño, con el propósito de regular los procesos de enseñanza y aprendizaje en diseño. Esta postura busca el fomento y desarrollo de habilidades cognitivas propias en diseño para conocer, analizar y comprender la realidad desde una óptica que sitúa al diseñador como un agente de cambio social.

En ese sentido, en el año 2019 un grupo de investigadores de los programas de Diseño e Ingeniería de Mercados de la Universidad, fueron convocados a desarrollar una acción diagnóstica de investigación en el corregimiento de Palermo, en el marco del proyecto institucional Modelo Alternativo de Inclusión e Innovación Productiva (MAIIP). Este modelo busca impulsar el desarrollo de los territorios a través del eje temático de mercadeo de lugares. A partir de esto se persigue la generación de estrategias de mercadeo para potenciar algunos aspectos culturales y productivos del territorio. Sin embargo, durante los acercamientos realizados a la comunidad se hizo evidente un desconocimiento de en discusión el desarrollo de proyectos de diseño con impacto social mediados por la virtualidad en los que los procesos de enseñanza sincrónicos y asincrónicos son protagonistas. De esta manera, se busca contribuir a la discusión disciplinar por medio de la relación de prácticas situadas en el desarrollo proyectual del diseño con el fin de que estas sean valoradas, adaptadas o resignificadas. Considera el semillero que así se podrá sensibilizar a los estudiantes sobre el desarrollo de posturas disciplinares propias que le den sentido a su acción profesional. El trabajo presentado, en primer lugar, aborda las relaciones entre los procesos de reflexión y acción en diseño mediante la metodología del doble diamante; segundo, aborda el uso de herramientas metodológicas y conceptuales como articuladores de procesos de pensamiento en diseño; tercero, se explica la estructura metodológica de doble diamante desarrollada por el semillero $3 \mathrm{E}$ y, finalmente, se relacionan unas estrategias didácticas sincrónicas y asincrónicas de trabajo colaborativo gracias a las que fue posible el proceso de investigación de manera remota.

El trabajo que aquí se presenta, inicialmente, aborda las relaciones entre investigación y creación dentro del proceso formativo de todo diseñador. Luego, se relacionan unas estrategias didácticas de acción-reflexión que buscan contribuir a la construcción social del 
conocimiento como forma de aprendizaje y, finalmente, se relaciona una metodología de investigación desde el diseño (doble diamante) a partir del abordaje de herramientas metodológicas y conceptuales modificadas en pro de organizar, clasificar y sistematizar información. Lo anterior con el fin de que a su vez se transformen en conocimiento transferible a una situación de diseño a intervenir.

\section{Metodología}

A partir de los desafíos producto de la pandemia mundial iniciada en el 2019 y el cambio de las dinámicas de aprendizaje e investigación la nueva apuesta desde el Semillero 3E tiene como finalidad entender y adaptar metodologías desde el pensamiento de diseño para que estas optimicen y procesen de forma ágil la información y su posterior corroboración de la misma.

Al afrontar los nuevos escenarios en donde las restricciones de interacción física limitan el acercamiento a la población, el semillero se vio en medio de una disyuntiva que se puede expresar de la siguiente manera. El pensamiento de diseño fue una de las bases teóricas y metodológicas del semillero. No obstante, una de sus principales consideraciones se rige bajo la idea de que la interacción con el contexto y las personas es de vital importancia para entender desde el diseño sus interacciones y complejidades. Dicha disyuntiva, ha conducido al grupo de investigación del Semillero 3E a participar confrontar y co-crear nuevos métodos, metodologías y herramientas que estén en pro de una investigación a distancia asertiva con la misma efectividad de una investigación de modo presencial.

Es por eso que el Semillero 3E tomó como línea de acción la pregunta de investigación: ¿Cómo incentivar la apropiación del patrimonio cultural en la comunidad del corregimiento de Palermo, Paipa? Esto con el objetivo de orientar un impacto social desde la disciplina del diseño que fomente la apropiación del patrimonio cultural del corregimiento de Palermo entre sus pobladores. Para lograr esto, se implementó el uso de dos grandes pilares transversales: Reflexión (exploración y mapeo) y acción (ideación). Ambos estructuran ocho fases de ejercicio investigativo (interpretar, comprender, delimitar, conceptualizar, valorar, idear, determinar y solucionar) (ver Figura 1).

Estas ocho fases permitieron sistematizar a partir de la construcción y adaptación de herramientas de diseño del design thinking (Ken y
O'Grady, 2018) al proceso de investigación con el fin de generar y sistematizar datos e información del proyecto y, asimismo, generar interpretaciones proyectuales propias del diseño. Para esto se aplicó

la metodología del doble diamante, dado que articula la exploración iterativa de diferentes instancias de pensamiento divergente y pensamiento convergente. De esta manera se construye un marco referencial en el que se abordan categorías conceptuales, tales como memoria, tejido social, patrimonio, capital sociocultural, identidad y apropiación. Estas últimas, hacen referencia a relaciones entre la memoria y la narración como elementos indispensables en todo proceso de construcción simbólica de la memoria. En este sentido la memoria es el insumo principal para producir un escenario de valoración patrimonial, donde los pobladores visualicen sus valores identitarios y a su vez su potencial de desarrollo.

Metodología del doble diamante en los procesos de acción y reflexión en diseño

Al asumir este reto que demanda la investigación de diseño de manera remota, uno de los puntos de partida fue la exploración y contraste de las diversas metodologías existentes de investigación en diseño. Entre las más importantes están: La metodología del doble diamante del Design Council (Ken y O'Grady, 2018; Design Council, 2015), human centered design de Mike Cooley (IDEO, 2018), systemic design de Systemic Design Research Network, el modelo de la escalera danesa de diseño del Design Council (Paredes López, Manrique y Carrillo, 2016), la metodología del design thinking de IDEO de Tim Brown (2008). Tal comparación se hizo con la finalidad de encontrar una metodología que mediara entre la búsqueda de información de forma eficaz, su síntesis y posterior evaluación a distancia.

Una de las variables que definieron el uso de la metodología del doble diamante para este caso de estudio con la comunidad de Palermo, fue la estimulación de los procesos de pensamiento divergentes y convergentes que puede obtener el diseñador mediante su quehacer profesional; es decir, aquella metodología que logre estimular el pensamiento divergente como lo explica Edward de Bono en su obra publicada en 1967 The Use of Lateral Thinking (De Bono, 1967). En ella, introduce el término de pensamiento lateral. De acuerdo con esto, para entender un problema y su solución, se debe ver las diferentes 
perspectivas de la incertidumbre para así poder encontrar diversas respuestas. Como lo aclara Buchanan (1992), en su artículo Wicked problems in design thinking, para problemas perversos, no existen soluciones definitivas ni mucho menos una secuencia de pasos en orden lineal. Por lo tanto, el uso de los procesos de pensamiento abierto y cerrado es ideal para visualizar los problemas de una forma sistemática; asimismo, se encuentra el pensamiento convergente, que, a su vez, como lo expone Joy Paul Guilford en 1997 en su modelo de inteligencia, es necesario una inteligencia convergente para poder consolidar un posible camino a la respuesta deseada.

Ahora bien, este pensamiento divergente y convergente que involucra la metodología del doble diamante, también influye en los procesos de toma de decisiones y postulados que el diseñador debe afrontar en todo el proyecto. En el artículo Experiencias en investigación acción-reflexión con educadores en proceso de formación en Colombia (Quintero Corzo, Muñoz Giraldo y Munévar Molina, 2002) el desarrollo de la investigación-acción ayuda al investigador en formación a comprender su indagación de forma colectiva, por lo que propone una práctica para desglosar el problema y así poder establecer una estrategia y conducir a un ejercicio de pensamiento deductivo. Ya con esta estrategia de acción el estado de reflexión se hace presente, puesto que pone a prueba y evaluación una hipótesis para poder iterar desde un pensamiento inductivo.

Por lo tanto, la formulación del doble diamante como metodología, permite introducir a los miembros del Semillero 3E en el desarrollo de un tipo de pensamiento creativo de forma divergente y convergente, en pro de la creación de soluciones con sentido de innovación e impacto en los aspectos políticos, económicos, sociales y culturales. En estos, su forma de diamante implica el uso del racionamiento en reflexión (divergente) en el momento de ver las diferentes aristas de un problema y el racionamiento en acción (convergente). De este modo, la metodología propone un foco de solución. Para que esto tenga sentido se debe aclarar que la metodología del doble diamante trabaja bajo dos grandes etapas. La primera se conoce como descubrir y entender el problema; la segunda, se llama desarrollar y entregar su solución. Ambas etapas están mediadas por un punto central nombrado el reto dentro del reto que permite evaluar la información e iterar.

\section{Resultados}

Investigar en diseño se ha convertido en un proceso en el que se miden, evalúan y reconocen los alcances profesionales y académicos de los diseñadores; al utilizar el diseño como sujeto, medio y fin para la investigación, en la producción de conocimiento (Jan Stappers y Giaccardi, 2010), la exploración de posibilidades para investigar no se limita únicamente a una ruta, ya que los procesos de diseño son dinámicos y deben estar en constante cambio para poder obtener buenos resultados en su ejecución.

Herramientas como articuladores de procesos de pensamiento En el momento del uso de herramientas y metodologías, se definen rutas y procesos con el fin de orientar la aplicación de conocimiento. De esta manera, posibilitan estructuras de pensamiento que garanticen la sistematización de información de manera clara, organizada y reconocible para el diseñador, con el fin de que esta pueda ser utilizada efectivamente en el desarrollo de propuestas de diseño. Las herramientas, dependiendo de su aplicación, transformación y posterior uso, dirigen los procesos de pensamiento divergente, ya que amplían la información y posibilitan la extensión en las consultas. Un ejemplo de ello, es la herramienta buzz report (Gasca y Zaragoza, 2018) y visualización de conceptos que condujo al Semillero $3 E$ a generar procesos de investigación- acción en torno a entender y buscar proyectos situados en la línea de memoria histórica, identidad y patrimonio. Por consiguiente, los procesos de pensamiento convergente e investigación en reflexión, que evalúan la calidad, eficacia y eficiencia de la información obtenida, se construyen mediante la elaboración de herramientas tales como conclusion buzz report y mapeo de conceptos, herramientas nuevas creadas por el Semillero 3E a raíz de la necesidad de síntesis e iteración de la investigación, finalizando con un Business Model Canvas (Tomitsch et al, 2021) con el objetivo de que los integrantes del Semillero evidenciaron posibles decisiones de diseño.

La apropiación de herramientas de diseño del proceso investigativo permite que estas mismas se acoplen de forma orgánica al proyecto, lo que hace que su aplicación sea próxima a las necesidades de la investigación, es decir, que si el investigador comprende el objetivo de la búsqueda de la información puede comprender el uso de la herramienta y su aplicabilidad. Es importante mencionar que 
las herramientas no necesariamente deben enfocarse únicamente en el entorno propuesto, sino que estas pueden ser modificadas y redireccionadas para obtener de forma más asertiva y puntual las exigencias del proyecto.

Por esa razón, herramientas como el Business Model Canvas (Osterwalder, y Pigneur, 2016), posibilitaron la evaluación de propuestas de diseño basadas en criterios de valor orientados para negocios innovadores. El buzz report organiza, jerarquiza, filtra y delimita fuentes y referentes de interés para el desarrollo del proyecto. Este, a su vez, trabaja en conjunto con la herramienta mapeo de conceptos, que ayudaron a clarificar el panorama de la investigación. La implementación de estas herramientas demuestra una variedad de formas para ser aplicadas y, al mismo tiempo, la necesidad de ser modificadas.

Basados en la experiencia del Semillero 3E la posibilidad de creación de nuevas herramientas para solucionar necesidades puntuales del proyecto y las experiencias en la aplicación de estas, dan como resultado una herramienta única del semillero 3E: el mapeo de conceptos, tiene la finalidad de relacionar, jerarquizar y sintetizar los conceptos que pueden llegar a redundar en el desarrollo del proyecto. En el proyecto de Palermo, esta herramienta fue útil para poder comprender, filtrar y depurar 26 conceptos sueltos. El ejercicio tuvo como resultado la síntesis de 12 conceptos claros con la finalidad de aplicarlos de forma asertiva en el desarrollo del proyecto. De estos 12, se identificaron 5 conceptos útiles para la propuesta de diseño (capital sociocultural, patrimonio, tejido social, narrativa y memoria).

El doble diamante aplicado a la apropiación del patrimonio cultural. Lo anterior, da lugar al desarrollo de una estructura metodológica que hace transitar los procesos de investigación en una constante divergencia y convergencia que incentivan la iteración, el cambio y la innovación. Semejante proceso se construye, como ya se mencionó con anterioridad, sobre los pilares de reflexión y acción y que a su vez se acercan a la primera etapa de la metodología del doble diamante, esto es, descubrir y definir (Design Council, 2015). Etapa en la cual se encuentra actualmente el semillero 3E. Esta, orienta al desarrollo de un esquema básico de diseño que posteriormente induce a la bocetación y prototipado de propuestas en la que se irán clarificando posibles decisiones y rutas productivas que se desarrollarán a lo largo de la segunda etapa de la metodología, que se traduce en desarrollar y entregar (Design Council, 2015).

El comportamiento de la investigación requirió la apertura de información para lograr entender el estado del proyecto y la situación del corregimiento de Palermo. De esta manera, la implementación de herramientas que cumplen con las necesidades específicas en el proceso de investigación para la recolección de datos benefició la rápida y amplia obtención de la mayor información. En este sentido, resultaron de gran utilidad herramientas como el buzz report y el mapeo de conceptos que permitieron la obtención de referentes, significados y definiciones que amplían la percepción de la situación y una gran variedad de posibilidades de enfoques en los resultados. Posteriormente la dedicada selección de información pertinente para el desarrollo del proyecto fue ejecutada a través de las herramientas buzz report (modificado por el semillero), mapeo de conceptos y Business Model Canvas. Gracias a ello, se lograron clarificar los criterios de diseño que en el proyecto de Palermo definieron la ruta de trabajo a través de conceptos articuladores que buscan dar respuesta a la necesidad de incentivar el patrimonio y sobre las cuales se basan las propuestas de diseño. Estos conceptos buscan estimular un sentido de pertenencia por el territorio, fortalecer los vínculos de los habitantes del corregimiento y promover el crecimiento productivo y económico del mismo a través del mercadeo de lugares enfocado en la identidad del territorio. El resultado del uso de estas herramientas fortaleció el dominio de los conceptos y, en consecuencia, la comunicación asertiva entre los integrantes del semillero, lo que facilitó el trabajo a través de la modalidad remota. Así pues, esta constante divergencia y convergencia en los procesos de investigación sondean las decisiones ya existentes en el proceso de diseño y dirigen las posteriores acciones en criterios de continuidad o replanteamiento de los mismos con el fin de lograr un resultado referente a las necesidades de la población.

\section{Conclusiones}

El diseño está llamado a reconocer en los diálogos interdisciplinares y transdisciplinares, una base de reflexión disciplinar. A partir de la misma, la investigación debe dar apertura a otras formas de conocimiento que consolidan las prácticas propias empleadas para el desarrollo de un proyecto. Más aún, cuando la relación entre 
investigación y diseño parece un tanto difusa dentro del ámbito académico y profesional, dada la amplia variedad de concepciones y posturas sobre el proceder metodológico a implementar.

Por ello, en el campo del diseño, los métodos, metodologías y herramientas no deben ser consideradas como aspectos modales o de tendencia en la investigación. Esto se debe a que por medio de su uso y modificación dichas herramientas deben adecuarse a las necesidades de sistematización y correlación de información en un proyecto, puesto que las herramientas normalmente no trabajan de manera exacta en todos los espacios de indagación. Condición que sitúa a la investigación en diseño como una oportunidad para consolidar el corpus teórico disciplinar y generar conocimiento susceptible de ser generalizado y aplicado a otros procesos o proyectos de diseño. De ahí que sea tan importante fortalecer los procesos de investigación formativa en diseño, no solo como una estrategia para comprender cómo explorar y reflexionar soluciones a problemas situados, sino como un laboratorio de experimentación abierta que fomente dinámicas flexibles de interrelación entre la práctica y el objeto de estudio del diseño. En consecuencia, los procesos de investigación formativa en diseño son una estrategia para abordar el conocimiento de una manera más flexible, en la que su principal insumo sean ideas disruptivas que fomenten el desarrollo de nuevas estructuras de pensamiento y métodos de diseño, en las que se generen escenarios de actuación disciplinar mediados por la investigación y, a su vez, estimulen la creatividad y la innovación desde una perspectiva proyectual.

La contingencia sanitaria Covid-19 obligó a la modificación de las dinámicas de trabajo y aprendizaje a nivel mundial incluyendo los procesos de investigación en diseño. De este modo, los procesos de investigación se trasladaron a entornos virtuales, lo que hizo que las modalidades de trabajo se adaptaran a los diferentes entornos donde se desarrollaban. Esta situación en donde el contacto físico impide la comunicación hace necesaria la preparación de las temáticas y apoyos para el desarrollo de los trabajos colectivos. Lo cual demandó nuevas dinámicas de aprendizaje que hicieron transitar los procesos de investigación presencial, a uno remoto lidiado por plataformas digitales y encuentros virtuales desde la sincronía (trabajo simultáneo) y la asincronía (trabajo autónomo).
Lo ideal sería integrar estos dos modelos adecuadamente con el fin de que resulte beneficioso para los integrantes en un proceso investigativo de diseño, con herramientas, plataformas y estrategias de carácter disruptivo. A partir de ello, se espera impulsar la participación activa y colaboración, para que el resultado sea equivalente o mejorado al de un encuentro presencial. No obstante, en el proceso de encontrar la técnica adecuada de trabajo, como diseñadores y miembros del Semillero 3E, se plantearon nuevas experiencias de aprendizaje funcionales que se encaminaron a lograr objetivos grupales significativos, superando. Así, se superaron los obstáculos técnicos y humanos que se iban presentando en el camino dando continuidad al crecimiento de habilidades y conocimientos investigativos.

Lo mencionado anteriormente permitió incorporar y fortalecer el intercambio metodológico interdisciplinar y adaptar estrategias de investigación cualitativa online de otras disciplinas (antropología, puntualmente etnografía) al proyecto, es decir, emplear instrumentos digitales para tener acercamientos a la comunidad de Palermo, entender su contexto de manera empática, al explorar contenido audiovisual, material recolectado en otras investigaciones y tener hallazgos significativos en el ciberespacio para comprender las prácticas, relaciones, emociones y hábitos de las personas. Todos los datos encontrados fueron analizados y filtrados con el fin de enriquecer y robustecer el conocimiento acerca de la comunidad, conocer sus valores identitarios y el patrimonio material e inmaterial para alcanzar un nivel reflexivo mucho más profundo y crear una estrategia de diseño alineada a sus intereses, expectativas y necesidades. Todo lo anterior con el objetivo de promover el rol del diseño no solo como una práctica centrada en la configuración de objetos, sino que va más allá como un agente social de cambio centrado en las personas (López-Macías, López-León y MollenhauerGajardo, 2020)

Sumado a lo anterior, las experiencias sincrónicas lograron generar cercanía como grupo y potenciar procesos de debate, opinión, reflexión e ideación, ya que estas estimulan la conversación en tiempo real (Vlásica, 2020) y respuesta inmediata entre diseñadores integrantes del Semillero 3E, lo que permite despejar dudas. El uso de plataformas digitales, como Microsoft Teams, facilitó de manera efectiva la reunión y comunicación para plantear objetivos y potencializar los alcances 
de la investigación. En estas sesiones generalmente se tocaban temas para sincronizar la información que se recogía del corregimiento y su comunidad, exponer los diferentes puntos de vista respecto a los avances logrados, presentar material de apoyo como plantillas y lecturas complementarias, y discusiones en tiempo real para llegar a conceptos colectivos que plantean una ruta a seguir

Adicionalmente, herramientas de libre acceso como MIRO, favorecieron sesiones grupales sincrónicas y asincrónicas de ideación -creación, de manera visual, interactiva y colaborativa. Estas sesiones cumplían el objetivo de generar escenarios de aprendizaje y creación entre los integrantes del Semillero 3E, para construir conocimiento colectivo a través de plantillas y pizarras para el desarrollo de journey map de usuarios, brainstorming, herramientas de experimentación y actividades de pensamiento divergente. Por último, se puede decir que compartir con pares genera mayores aprendizajes, debido a que se cuestiona los conceptos aprendidos, se desarrollan nuevas habilidades y destrezas que permiten involucrarse en el contexto de la investigación.

Factor que contrasta con el aprendizaje asincrónico que estimula la creación de conocimiento autónomo, autogestión y compromiso en los participantes del Semillero 3E, ya que este permite mediar su atención, tiempo, recursos y objetivos preestablecidos. De este modo, contribuye a construir procesos de investigación paralelos. En este caso, el uso de SharePoint, como nube de contenido que almacena lecturas, contenido audiovisual y plantillas de las herramientas de diseño para su posterior desarrollo en la investigación. Esta plataforma permite el trabajo diferido de cada uno de los investigadores, lo que fomenta su responsabilidad individual con el trabajo de equipo para posteriormente socializar estos resultados en los encuentros sincrónicos.

Por ello en el campo del diseño, el proyecto de Palermo, como caso de estudio sirvió de plataforma para generar una experiencia de aprendizaje colaborativo en diseño, que comprueba cómo los procesos de investigación mediados por la virtualidad, viabilizan la estructuración de un sistema temporal eficiente para la explicación y sustentación de conceptos, la planeación y alcance de objetivos, el desarrollo de dinámicas de trabajo colaborativo remoto, y la lectura empática de las condicionantes en un contexto. Mediante el desarrollo de herramientas de diseño que dinamicen la consecución de resultados eficaces en condiciones de emergencia sanitaria.
En conclusión, el proyecto de Palermo como caso de estudio sirvió de plataforma para generar una experiencia de aprendizaje colaborativo en diseño, donde se comprueba que los procesos de investigación mediados por la virtualidad viabilizan la estructuración de un sistema temporal eficiente para la explicación de conceptos, sustentación de ideas, planeación de objetivos y desarrollo de dinámicas de trabajo colaborativo remoto. Ahora por parte del uso de las metodologías y herramientas de diseño, la dinámica es totalmente diferente a un proceso presencial, puesto que el acceso a la información, empatizar en contexto, prototipar las ideas y testear la solución han sido de difícil desarrollo puesto que las condicionantes de la emergencia sanitaria, no se logran comprobar en tiempo real diversas hipótesis que se logran proponer en los procesos sincrónicos y asincrónicos debatidos en el aula.

\section{Referencias bibliográficas}

Brown, T. (Junio de 2008). Design Thinking. Harvard Business Review. Recuperado de: https://readings.design/PDF/Tim\%20Brown,\%20 Design\%20Thinking.pdf

Buchanan, R. (1992). Wicked Problems in Design Thinking. Design Issues, $8(2), 5-21$.

Ken, J. y O'Grady, V. (2018). Manual de Investigación para diseñadores. Barcelona: Blume.

De Bono, E. (1967). The Use of Lateral Thinking. Barcelona: Paidós

Design Council. (2015). Design methods for developing services. Recuperado de: https://www.designcouncil.org.uk/sites/ default/files/asset/document/Design\%20methods\%20for\%20 developing\%20services.pdf.

Brown, T. (2020). Diseñar el cambio. Barcelona, España: Empresa Activa. IDEO. (2018). Design Kit: The Field Guide to Human-Centered Design Toolkit. California: IDEO.

Gasca, J. \& Zaragozá, R. (2018). Buzz Report de Designpedia. Madrid: Lid Editorial.

López-Macías, G. A., López-León, R. A. \& Mollenhauer-Gajardo, K. (2020) Etnodiseño: Modelo de diseño desde la perspectiva etnográfica y su aporte a la formación en diseño, i+Diseño. Revista científicoacadémica Internacional de innovación, investigación y desarrollo en Diseño, 15, 23-40. DOI: https://doi.org/10.24310//diseno.2020. v15i0.9574 
Osterwalder, A. \& Pigneur, Y. (2016). Generación de Modelos de Negocio.

Barcelona: Deusto.

Paredes López, S. P., Manrique, A. \& Carrillo, N. (2016). Checking design.

Bogotá: Editorial Pontificia Universidad Javeriana.

Quintero Corzo, J., Muñoz Giraldo, J. F. \& Munévar Molina, R. A. (2002).

Experiencias en investigación-acción-reflexión con educadores

en proceso de formación en Colombia. Revista Electrónica de Investigación Educativa, 4(1), 66-80.

Tomitsch, M., Borthwick, M., Ahmadpour, N., Cooper, C., Frawley, J., Hepburn, L.A., Kocaballi, A.B., Loke, L., Núñez-Pacheco, C., Straker, K. \& Wrigley, C. (2021). Design. Think. Make. Break. Repeat. A Handbook of Methods. Amsterdam: Bis Publishers.

Vlásica, J. (Abril de 2020). Lo síncrono y lo asíncrono: cómo diseñar una sesión online. Innovación Educativa. Recuperado de: https:// innovacioneducativa.upc.edu.pe/2020/04/14/como-disenar-uncurso-online-definicion-de-sesion-online-lo-sincrono-y-lo-asincrono/ 